Divers@ Revista Eletrônica Interdisciplinar,

Matinhos, v. 13, n. 2, p. 164-173, jul./dez. 2020

ISSN 1983-8921

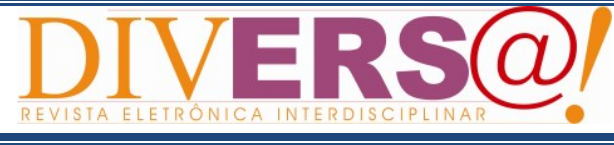

\title{
DISTRIBUIÇÃO ESPACIAL DA COVID-19 EM EUNÁPOLIS-BA
}

\section{SPATIAL DISTRIBUTION OF COVID-19 IN EUNÁPOLIS-BA}

Jeorge Luis Martins Oliveira ${ }^{1}$

Nereide Santos Lisboa ${ }^{2}$

Elfany Reis do Nascimento Lopes ${ }^{3}$

\begin{abstract}
Resumo
A pandemia da COVID-19, ocasionada pelo novo vírus da família coronavírus (SARS-CoV-2) já pode ser considerado o maior desafio da saúde pública do século XXI. O presente artigo teve como objetivo avaliar a distribuição espacial dos casos e óbitos confirmados de COVID-19 de Eunápolis, visando compreender a disseminação do vírus em uma cidade margeada por uma rodovia federal e via transitória de acesso para diversas cidades do Sul da Bahia e Minas Gerais. Foi realizado pesquisa descritiva e de análise exploratória dos casos de COVID-19 entre os meses de março a julho. Calcularam-se as taxas de incidência, mortalidade e letalidade. Os dados foram extraídos de bases governamentais epidemiológicas municipais e estaduais e quantificadas as taxas de casos confirmados e óbitos, além da espacialização utilizando o sistema de informações geográficas QGIS. Foram registrados 2.068 casos e 34 óbitos, sendo $53,77 \%$ dos casos em sexo feminino e $70,59 \%$ dos casos no sexo masculino. Indivíduos na faixa de 30 a 49 anos foram mais prevalentes entre os casos, enquanto indivíduos de $\geq 80$ anos prevaleceram com a maior frequência entre os óbitos. A maior incidência foi observada no mês de julho (10,09 por 1 mil habitantes) e a maior letalidade, no mês de junho (2,53\%). Observou-se maior taxa de infecção e morte pela doença em bairros como Pequi, Dinah Borges e Centro. Agravos em indivíduos masculinos são prevalentes mesmo com taxas elevadas em mulheres, induzindo a necessidade de investigação de comorbidades, fisiologia do sistema respiratório e imunológico da população.
\end{abstract}

Palavras-chave: Pandemia; infecção; Análise Espacial; Epidemiologia.

\begin{abstract}
The COVID-19 pandemic caused by the new coronavirus family virus (SARS-CoV-2) can already be considered the greatest public health challenge of the 21st century. This article aimed to evaluate the spatial distribution of confirmed cases and deaths of COVID-19 in Eunápolis, aiming to understand the spread of the virus in a city surrounded by a federal highway and transitory access to several cities in the south of Bahia and Minas Gerais General. Descriptive research and exploratory analysis of COVID19 cases was carried out between March and July. Incidence, mortality and lethality rates were calculated. Data were extracted from municipal and state epidemiological government databases and the
\end{abstract}

\footnotetext{
Artigo Original: Recebido em 20/09/2020 - Aprovado em 13/10/2020.

${ }^{1}$ Mestrando em Ciências e Tecnologias Ambientais pela Universidade Federal do Sul da Bahia / Instituto Federal da Bahia, Docente da rede privada e pública do estadoda Bahia.e-mail: jeorge_martins@yahoo.com.br (autor correspondente)

${ }^{2}$ Doutoranda em Pesquisa Clínica em Doenças Infecciosas pelo Instituto Nacional de Infectologia Evandro Chagas-Fiocruz, bióloga da vigilância epidemiologica do municipio de Teixeira de Freitas. e-mail: nereidelisboa@gmail.com

${ }^{3}$ Doutor em Ciências Ambientais pela Universidade Estadual Paulista Júlio de Mesquita Filho / Instituto de Ciência e Tecnologia de Sorocaba e do Instituto de Pesquisas Tecnológicas de São Paulo (IPT). Docente da Universidade Federal do Sul da Bahia.e-mail:elfany@csc.ufsb.edu.br
} 
rates of confirmed cases and deaths were quantified, in addition to spatialization using the geographic information system QGIS. 2,068 cases and 34 deaths were recorded, with 53.77\% of cases in females and $70.59 \%$ of cases in males. Individuals aged 30 to 49 years were more prevalent among cases, while individuals aged $\geq 80$ years prevailed more frequently among deaths. The highest incidence was observed in July (10.09 per 1,000 inhabitants) and the highest lethality, in June (2.53\%). A higher rate of infection and death from the disease was observed in neighborhoods such as Pequi, Dinah Borges and Centro. Diseases in male individuals are prevalent even with high rates in women, inducing the need to investigate comorbidities, physiology of the respiratory and immune systems of the population.

Keywords: Pandemic; infection; Spatial Analysis; Epidemiology.

\section{Introdução}

Os surtos de gripe estiveram presentes em diversos momentos das civilizações e assumiam diferentes feições de transmissibilidade e letalidade conforme contexto sociocultural e econômico que estavam inseridos. Contudo, a pandemia da COVID19, ocasionada pelo novo vírus da família coronavírus (SARS-CoV-2) já pode ser considerado o maior desafio da saúde pública do século XXI (AUERBACH; OSELAME; DUTRA, 2014). Se tornou a maior crise global de saúde desde a propagação da gripe espanhola no início do século $\mathrm{XX}$, que acometeu cerca de $30 \%$ da população mundial da época, dizimando mais de 40 milhões de vidas (CAMPOS, 2005).

Trata-se de um vírus de origem animal que conseguiu evoluir ao ponto de infectar humanos, provavelmente através do contato com animais silvestres utilizados para alimentação (THE NOVEL, 2020). Acredita-se que a primeira transmissão tenha ocorrido em um mercado ou feira ao ar livre nas imediações da cidade de Wuhan, na China Central, no início de dezembro de 2019 e relatada ao escritório da Organização Mundial da Saúde (OMS), também na China, em 31 de dezembro de 2019.

$\mathrm{O}$ vírus se expandiu rapidamente para todo o mundo e, em janeiro de 2020, o governo chinês notificou a Organização Mundial da Saúde (OMS) informando que o patógeno causador do surto havia sido identificado como um novo coronavírus (SARS-Co-V2) (WU; MCGOOGAN, 2020). Em março de 2020, a COVID-19 foi caracterizada pela OMS como uma pandemia, devido à rápida disseminação dos vírus da síndrome respiratória aguda grave coronavírus 2 (SARS-CoV-2)
(CASTRO et al., 2020). De acordo com a Johns Hopkins University \& Medicine (2020), em junho de 2020, já havia sido confirmado um número superior a 10 milhões de infectados e mais de 500 mil óbitos.

Muitos fatores podem definir o percurso de uma pandemia, alguns dos quais são pouco conhecidos atualmente para o COVID-19 (ANDERSON et al., 2020). A associação entre a elevada capacidade de infecção do patógeno e uma população vulnerável que ocupa áreas excessivamente diversificadas em relação às condições socioeconômicas e um sistema de saúde com limitações, tem cooperado para um cenário de risco diferenciado para ocorrência da COVID-19 (SANTOS et al., 2020; NASCIMENTO; PACHECO, 2020).

O escasso conhecimento científico no que diz respeito a distribuição geográfica e o elevado poder de disseminação dessa doença, geram incertezas sobre quais seriam as melhores estratégias a serem utilizadas para o enfrentamento da pandemia em diferentes partes do mundo (WERNECK; CARVALHO, 2020). Além da alta capacidade biológica de contágio do coronavírus, Oliveira Neto, Garcia e Spinussi (2020) afirmam que a sua rápida disseminação se deu pela "intensa fluidez e capilaridade da circulação mundial", seja pela facilidade de deslocamento entre cidades e países, pela ausência de higiene básica ou, ainda, pela facilidade de permanência do vírus em superfícies diversas.

No Brasil, os desafios são ainda maiores, pois as características de transmissão da COVID-19 em um país tropical, com desigualdade social evidente, precariedade de saneamento básico, e ausência de planejamento urbano com intensas áreas de aglomeração e aparente dificuldade de isolamento 
social ainda estão em investigação. Até o dia 19 de agosto foram registrados no Brasil 4.528.240 casos e 136.532 óbitos por COVID-19 (BRASIL, 2020a).

A situação apresentada pelo Brasil, considerado o epicentro da doença nas Américas, reforça a condição de Beck (1992), quando expôs que a sociedade contemporânea pode ser traduzida como "a sociedade de risco", pois a distribuição do risco não considera as assimetrias sociais, econômicas ou geográficas. $\mathrm{O}$ risco tornou-se generalizado no espaço e no tempo, não obedecendo fronteiras físicas ou a um espaço estático e imutável. A geografia como área de estudo da compreensão do fenômeno regional contribui para o entendimento e a análise das representações que assolam as cidades (MESQUITA et al., 2020).

A intrínseca relação entre a geografia e a epidemiologia, assegura que o uso de técnicas cartográficas e de geoprocessamento para a compreensão da organização do espaço, possibilitando aproximação dos fatores de desenvolvimento em grupos sociais com o monitoramento remoto de vastas áreas do espaço geográfico, fornecendo uma gama de informações fundamentais sobre as variáveis do risco (BONFIM; MEDEIROS, 2008; BUFFON; PAZ, 2018).

Consoante a necessidade de compreender a dinâmica de disseminação da COVID-19 no país, sobretudo no interior do Brasil, será essencial para estabelecer relações de causa e efeito, assim como a precariedade da estrutura sanitária e de saúde de um país extenso territorialmente, com diferentes culturas, economias e vulnerabilidades. Nessa condição que o estudo avalia a distribuição espacial dos casos e óbitos confirmados de COVID-19 de Eunápolis, visando compreender a disseminação do vírus em uma cidade atravessada por uma rodovia federal e via transitória de acesso para diversas cidades do Sul da Bahia e Minas Gerais. O estudo contribui com aspectos geográficos do arranjo espacial apresentado pela doença no interior do país, em um momento que se busca elucidar as características sociais e o impacto econômico da COVID-19, permitindo compreender a dinâmica da disseminação do coronavírus e a evolução da taxa de contaminação em bairros da cidade.

\section{Materiais e Métodos}

Situado na porção Sul do Estado da Bahia, no Território de Identidade da Costa do Descobrimento, o município de Eunápolis encontra-se entre as coordenadas geográficas $16^{\circ} 22^{\prime} 40^{\prime \prime} \mathrm{S}$ e $39^{\circ} 44^{\prime} 48^{\prime \prime} \mathrm{W}$, com altimetria média de 189 metros. A vegetação original é a Mata Atlântica e o clima é classificado como subúmido, apresentando temperatura média anual de $23,2^{\circ} \mathrm{C}$ e precipitação pluviométrica média de 1000 a $1200 \mathrm{~mm} / \mathrm{ano}$ (SEI, 2015). Possui uma área de $1.179,10 \mathrm{~km}^{2} \mathrm{e}$ densidade demográfica média de 84,97 habitantes $/ \mathrm{km}^{2}$, com população estimada de 114.396 habitantes em 2020 (IBGE, 2020).

A pesquisa é descritiva e de análise exploratória dos casos de COVID-19, cujas unidades de análise foram os 54 bairros pertencentes ao perímetro urbano e as diferentes áreas e distritos que compõem a zona rural do município. Eunápolis, assim como a grande maioria dos municípios brasileiros, possui desigualdades sociais perceptíveis, devido ao grande número de pessoas em pobreza extrema, que residem em bairros periféricos do município, e têm dificuldade de acesso a serviços de saúde de qualidade (JOLY, 2007).

Conforme a classificação de Regiões de Influência das Cidades (REGIC) pelo IBGE (2018), Eunápolis é uma Capital Regional C, exercendo e recebendo influência dos sobre os municípios baianos de Guaratinga, Itabela, Itagimirim, Itapebi, Porto Seguro, Santa Cruz Cabrália e Belmonte. Além disso, essa coinfluência também ocorre nos municípios mineiros de Salto da Divisa e Santo Antônio do Jacinto. Para fazer tal classificação, o IBGE leva em consideração as principais ligações de transportes regulares e os principais destinos dos moradores dos municípios supracitados para obter produtos e serviços, tais como compras em geral, educação superior, uso de aeroportos, serviços de saúde, bem como os deslocamentos para aquisição de insumos e o destino dos produtos agropecuários. Além disso, a crescente ampliação na oferta em cursos de ensino superior associado ao crescimento econômico local tem propiciado um aumento no fluxo migratório e consequentemente um aumento na população do município, visto que as rodovias federais BR-101 e BR-367 atravessam a cidade. Dessa forma, Eunápolis torna-se a principal rota de circulação para os demais municípios citados. 
Nesse estudo foram incluídos todos os casos e óbitos confirmados de COVID-19 do município entre os meses de março a julho de 2020. Foi construído um banco de dados espaciais com o levantamento de casos e óbitos confirmados de SARS-CoV-2, por cidade de residência, em bases de dados oficiais governamentais da Secretária de Saúde da Bahia (SESAB) (BAHIA, 2020a) e do sistema SIVEP-gripe, cedido pelo Núcleo Regional de Saúde (NRS-Extremo Sul), considerando as notificações presentes até o mês de setembro de 2020. O número de casos por bairros e por mês foram coletados a partir dos boletins descritos do município de Eunápolis, de acesso livre.

As variáveis estudadas foram sexo (feminino, masculino, sem informação), idade (média em anos), faixa etária ( 0 a 9,10 a 19, 20 a 29,30 a 39,40 a 49,
50 a 59, 60 a 69, 70 a 79, $\geq 80$, sem informação). As frequências absoluta e relativa de casos e óbitos foram descritas segundo sexo e faixa etária e bairro de residência. Foram calculadas as taxas de incidência e taxa de mortalidade por mil habitantes.

A base cartográfica municipal, com divisão por bairros com a delimitação do perímetro, bairros, zonas rural e urbana, foi adquirida junto à Secretaria Municipal de Infraestrutura (Figura 1). Para o desenvolvimento das análises espaciais utilizou-se o software livre QGIS versão 3.10 e para construção dos gráficos e tabelas foi utilizado Microsoft Excel.

$\mathrm{O}$ estudo respeitou as políticas que envolvem pesquisas em seres humanos e por se tratar de dados de domínio público, não foi submetido a um comitê de ética em pesquisa.

Figura 1 - Localização do município de Eunápolis, na Bahia, Brasil e a delimitação espacial dos bairros na área urbana

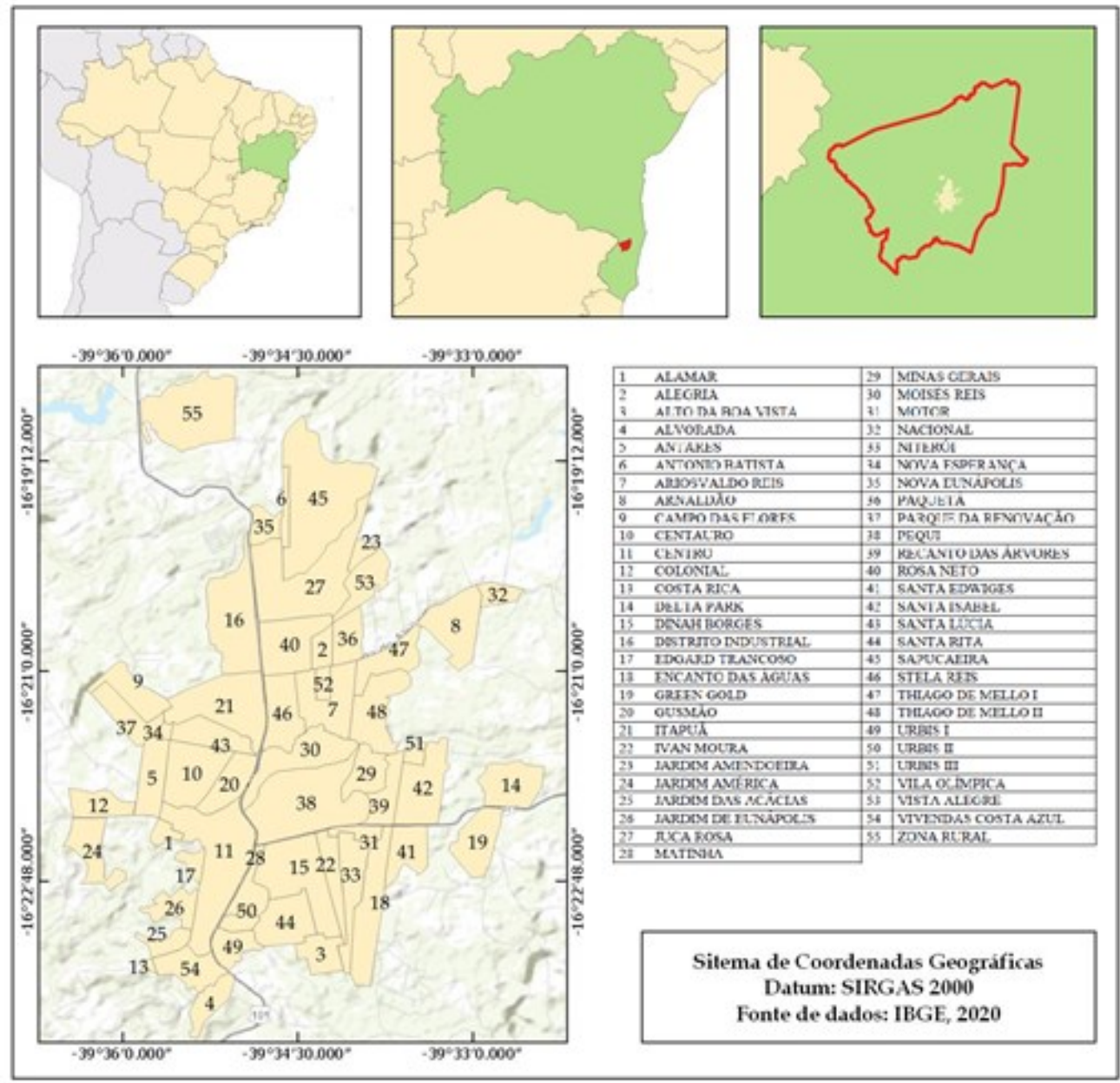

Fonte: Elaborado pelos autores a partir de IBGE (2020). 


\section{Resultados}

A evolução dos casos confirmados da COVID19 na cidade pode ser observada na Figura 2. Entre os meses de março a julho de 2020, foram registrados um total de 2.068 casos e 34 óbitos, partindo de apenas 1 caso no mês de março para 1.144 no mês de julho.

Figura 2 - Casos confirmados da COVID-19, no município doe Eunápolis, de março a julho de 2020 (N=2.068)

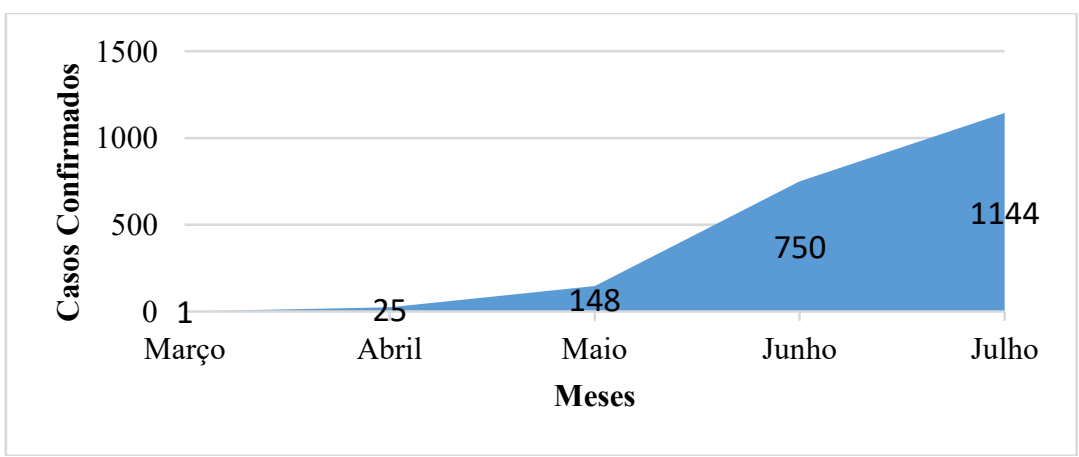

Fonte: Elaborado pelos autores a partir de SESAB / SIVEP-Gripe (2020).

Do total de casos, $46 \%$ eram do sexo masculino e $54 \%$ do sexo feminino, enquanto, que do total de óbitos, 70,59\% eram do sexo masculino, 29,41\% do sexo feminino. A maior taxa de incidência se deu no sexo feminino $(9,72)$ e a maior taxa de letalidade no sexo masculino $(2,53)$. Indivíduos na faixa de 30 a 49 anos foram mais prevalentes e tiveram uma maior taxa de incidência entre os casos, enquanto indivíduos de $\geq 80$ anos aparecem com a maior frequência e letalidade entre os óbitos. A Tabela 1 apresenta detalhadamente os quantitativos por faixa etária, com as respectivas taxas de incidência e letalidade.

Tabela 1 - Frequências absoluta, relativa de casos, taxa de incidência, óbitos e letalidade confirmados da COVID19, por sexo e idade, no município de Eunápolis, de março a julho de 2020

\begin{tabular}{|c|c|c|c|c|c|c|c|}
\hline \multicolumn{2}{|c|}{ Variáveis } & $\begin{array}{c}\text { Casos } \\
\text { (n) }\end{array}$ & $\begin{array}{c}\text { Casos } \\
(\%) \\
\end{array}$ & $\begin{array}{c}\text { Taxa de } \\
\text { incidência }\end{array}$ & $\begin{array}{c}\text { Óbitos } \\
\text { (n) }\end{array}$ & $\begin{array}{c}\text { Óbitos } \\
(\%)\end{array}$ & $\begin{array}{c}\text { Letalidade } \\
(\%)\end{array}$ \\
\hline \multirow{3}{*}{ Sexo } & Masculino & 950 & 45,94 & 8,30 & 24 & 70,59 & 2,53 \\
\hline & Feminino & 1112 & 53,77 & 9,72 & 10 & 29,41 & 0,90 \\
\hline & Sem informação & 6 & 0,29 & 0,05 & - & - & - \\
\hline \multirow{10}{*}{$\begin{array}{c}\text { Idade } \\
\text { (em anos) }\end{array}$} & 0 a 9 & 68 & 3,29 & 0,59 & 1 & 2,94 & 1,47 \\
\hline & 10 a 19 & 95 & 4,59 & 0,83 & - & - & - \\
\hline & 20 a 29 & 381 & 18,42 & 3,33 & - & - & - \\
\hline & 30 a 39 & 597 & 28,87 & 5,22 & 1 & 2,94 & 0,17 \\
\hline & 40 a 49 & 438 & 21,18 & 3,83 & 5 & 14,71 & 1,14 \\
\hline & 50 a 59 & 257 & 12,43 & 2,25 & 6 & 17,65 & 2,33 \\
\hline & 60 a 69 & 138 & 6,67 & 1,21 & 7 & 20,59 & 5,07 \\
\hline & 70 a 79 & 63 & 3,05 & 0,55 & 4 & 11,76 & 6,35 \\
\hline & $80+$ & 29 & 1,40 & 0,25 & 10 & 29,41 & 34,48 \\
\hline & Sem informação & 2 & 0,10 & 0,02 & - & - & - \\
\hline
\end{tabular}

Fonte: Elaborado pelos autores a partir de SESAB / SIVEP-Gripe (2020). 
Quando analisadas as taxas de incidência e letalidade por mês de ocorrência, a Figura 3 evidencia que a maior incidência foi observada no mês de julho e a maior letalidade, no mês de junho. A média de incidência foi de 18,08 (por 1 mil hab.) e letalidade $1,64 \%$.

Figura 3 - Taxa de incidência e letalidade dos casos de COVID-19, segundo o mês de ocorrência, Eunápolis, 2020

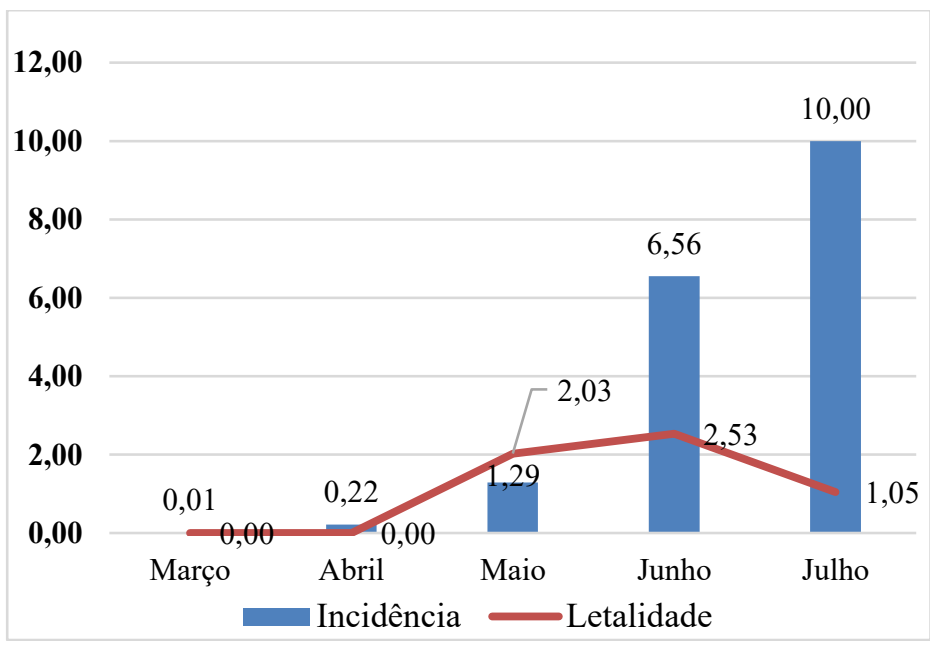

Fonte: Elaborado pelos autores a partir de SESAB / SIVEP-Gripe (2020).

O número de casos indicou o aumento progressivo da contaminação da população, aumentando de 25 para 47 (incremento de $88 \%$ ) entre maio e junho e para 51 no mês de julho (incremento de 9\%). Foi possível verificar um incremento de $360 \%$ de casos entre maio e junho e $139 \%$ entre junho e julho. Apenas 3 bairros não tiveram casos de COVID-19 no período. Os menores percentuais de casos ocorreram nos bairros Cajueiro (0,05\%), Ivan Moura (0,05\%), Delta Park $(0,14 \%)$ e Matinha $(0,14 \%)$ e os maiores no Centro, responsável por $8,09 \%$ do total de casos, Dinah Borges $(10,14 \%)$ e Pequi $(16,03 \%)$, conforme Figura 4.

Figura 4 - Distribuição espacial do número de casos confirmados acumulados por COVID-19 no município de Eunápolis, entre os meses de maio a julho de 2020

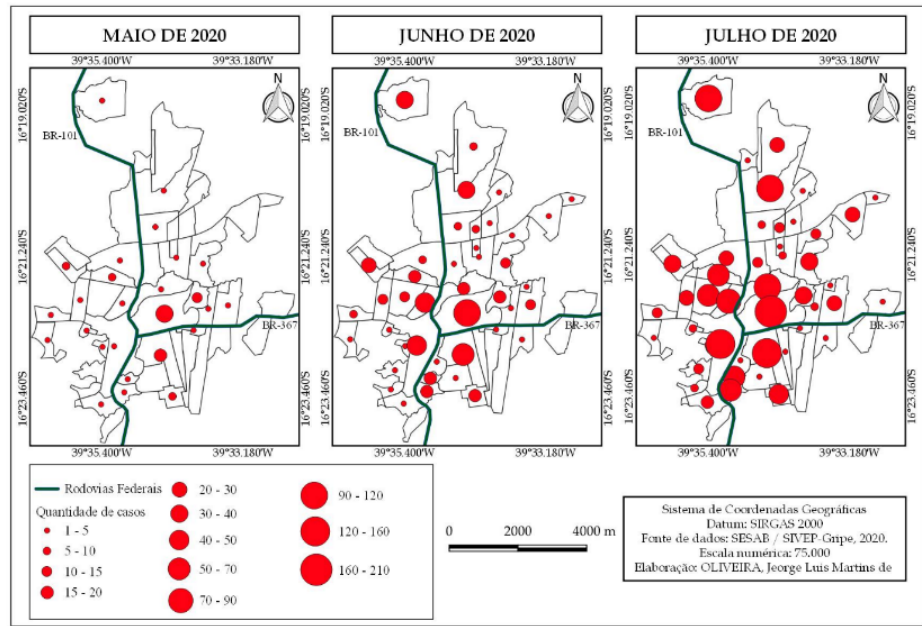

Fonte: Elaborado pelos autores a partir de SESAB / SIVEP-Gripe (2020). 
Na Figura 5, a distribuição mensal e acumulativo dos óbitos pode ser observada. Verificou-se que 3 bairros apresentaram registro no mês de maio, 10 no mês de junho e 11 no mês de julho. Dentre estes, o maior número ocorreu no centro como 4 óbitos
$(11,76 \%)$ e Pequi com $6(17,65 \%)$. O mês com maior número de óbitos foi junho com 19 (55,88\%) óbitos, seguido de julho com $12(35,29 \%)$ e maio $\operatorname{com} 3(8,82 \%)$.

Figura 5 - Distribuição espacial do número de óbitos confirmados por COVID-19 no município de Eunápolis, entre os meses de maio a julho de 2020

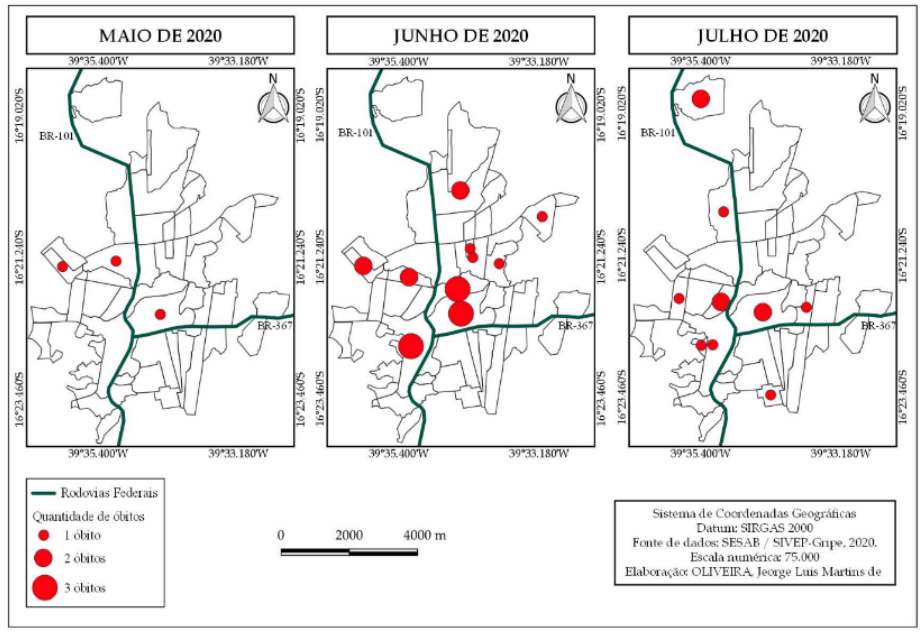

Fonte: Elaborado pelos autores a partir de SESAB / SIVEP-Gripe (2020).

\section{Discussão}

Este estudo analisou a distribuição espacial da COVID-19 no município de Eunápolis. A medida de incidência encontrada para o município $(18,08 / 1000$ habitantes) foi maior que a estadual $(11,17 / 1000$ habitantes), enquanto que a de letalidade foi menor (BAHIA, 2020b). Os dados de mortalidade encontrados seguem o padrão dos dados estaduais e nacionais, com maior risco de óbito para pessoas do sexo masculino e maiores de 60 anos (BRASIL, 2020b; BAHIA, 2020b).

Diferentemente do perfil nacional, mas corroborando com os dados estaduais, a contaminação predominou entre indivíduos do sexo feminino. Em relação a faixa etária mais acometida houve também uma similaridade com padrão estadual, predominando os números de casos em adultos jovens (BAHIA, 2020b. Padrões semelhantes foram encontrados no estudo de Cavalcante e Abreu (2020) indicando que a COVID-19 infecta um número maior de pessoas economicamente ativas.
Em relação aos óbitos, ainda seguindo a tendência nacional e estadual, eles foram mais comuns entre o sexo masculino e idosos, pessoas com doenças crônicas e indivíduos com histórico de imunossupressão. Biomarcadores de inflamação, como a proteína $\mathrm{C}$ reativa (CRP) e ferritina, tiveram aumento especialmente em homens mais velhos com COVID-19, enquanto outros marcadores, como testes de função hepática anormais eram comuns em várias faixas etárias, exceto para jovens e mulheres. Basófilos e eosinófilos do sangue periférico baixo também foram mais comuns no idosos com COVID19 (TEN-CATEN et al., 2020).

Em relação ao aumento do número de infectados, isso deve tanto pelo aumento real no número de casos, quanto pela ampliação da testagem de 100\% dos casos de Síndrome Gripal (SG) nas unidades públicas de saúde que acorrem a partir do mês de junho. Anteriormente, apenas de grupos específicos preconizados pelo Ministério da Saúde eram testados (BRASIL, 2020c).

A cidade de Eunápolis é um importante ponto de entrada tanto por abrigar um grande fluxo de comércio e empresas, quanto para viajantes, já que é 
cortada pela BR 101 e também fica a apenas $64 \mathrm{~km}$ de Porto Seguro onde encontra-se um aeroporto internacional. Os primeiros registros de infectados foram de dois homens que haviam retornado de uma viagem de trabalho a uma cidade da região com sintomas gripais.

A partir da distribuição espacial do número de casos no município, foi possível verificar importantes incrementos no decorrer dos meses no que diz respeito aos números casos positivos e bairros com casos positivos. Esse fato denota mais uma vez que a dinâmica econômica e social de alta conectividade entre os bairros, o que favorece sua transformação num polo de disseminação da doença para outros territórios e reforça a necessidade de compreender o padrão de transmissão da doença, para melhor implementação de medidas de mitigação e de controle (KRITSKI et al., 2020).

Os bairros Pequi, Dinah Borges e Centro foram os mais afetados pela doença. Vale ressaltar que esses bairros são de grande extensão territorial e extremamente populosos e com exceção do bairro Centro, os outros dois são bairros considerados de classe média-baixa e classe média, respectivamente. Além disso, ambos possuem um comércio intenso e o bairro Pequi ainda abriga uma das feiras livres mais tradicionais da cidade, o que pode ter contribuído para a disseminação da doença, já que muitas medidas de segurança e isolamentoo foram ignoradas por parte da população e comerciantes. Associada a esse comportamento tem-se ainda a ocorrência numa população completamente suscetível ocupando territórios extremamente heterogêneos quanto às condições de vida, o que aponta para o risco diferenciado de ocorrência da COVID-19 (SANTOS et al., 2020).

Com relação ao número de óbitos por bairro, este seguiu uma tendência parecida do número de casos, sendo o bairro do Pequi e Centro mais afetados, respectivamente. Tal fato pode estar ligado as dificuldades enfrentadas por essa população ao acesso à rede de serviços de saúde, além do comportamento socioeconômico e cultural da população (CAVALCANTE; ABREU, 2020).

\section{Conclusão}

Este estudo avaliou a distribuição espacial dos primeiros casos e óbitos pela COVID-19 no município de Eunápolis. Os resultados das análises mostraram maior quantidade de casos de infecção pela doença em bairros como Pequi, Dinah Borges e Centro e morte nos bairros Pequi e Centro. Essa distribuição possa se alterar rapidamente, devido à elevada capacidade de transmissão da COVID-19, aliada às diferenças sociais entre os bairros de Eunápolis. Novos estudos que empreguem análises espaciais dos óbitos e casos recuperados da doença irão contribuir para se compreender a evolução da COVID-19 no município. Analisar o perfil espacial de uma nova doença no território é essencial para a compreensão de padrões e adoção de ações de controle. Sugere-se estudos mais profundos e planos emergenciais para a COVID-19 que considerem as características socioeconômicas e culturais dos bairros presentes no território, a fim de se alcançar uma maior efetividade com as medidas de controle.

Por fim, vale ressaltar que este é um estudo preliminar e engloba apenas um determinado período amostral, não representando uma análise completa da disseminação da pandemia no município. No entanto, essa pesquisa e suas análises podem servir como base para futuras discussões sobre o tema. Além disso, a metodologia proposta demonstra a importância do geoprocessamento e a geografia, em especial a geografia do risco, para o conhecimento e disseminação da doença no município além de servir como ferramenta de gestão e base para decisões de alocação de equipamentos de saúde.

\section{Referências}

ANDERSON, R. M.; HEESTERBEEK, H.; KLINKENBERG, D.; HOLLINGSWORTH, T. D. How will country-based mitigation measures influence the course of the COVID-19 epidemic? The Lancet, v. 395, n. 10228, p. 931-934, mar. 2020 .

AUERBACH, P.; OSELAME, G. B.; DUTRA, D. A. Revisão Histórica da Gripe no mundo e a nova H7N9. Revista de Medicina e Saúde de Brasília, v. 2, n. 3, p. 183-197, 2014

BAHIA. Secretária de Saúde da Bahia - SESAB. Central Integrada de Comando e Controle de 


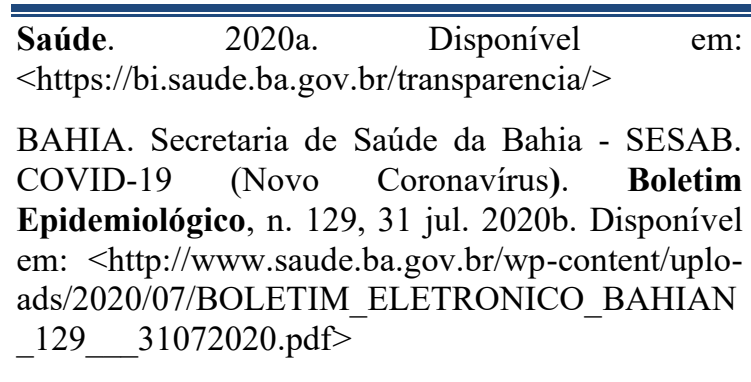

BECK, U. Risk Society. Towards a new modernity. Londres: Sage Publications, 1992.

BONFIM, C.; MEDEIROS, Z. Epidemiologia e geografia: dos primórdios ao geoprocessamento. Espac. Saúde, v.10, n.1, p.53-62, 2008.

BRASIL. MINISTÉRIO DA SAÚDE. COVID-19: Painel Coronavírus. 2020a. Disponível em $<$ https://covid.saude.gov.br/>

BRASIL. MINISTÉRIO DA SAÚDE. Centro de Operações de Emergência em Saúde Pública. Coronavírus COVID-19, 10 de abril de 2020. Bol Epidemiol Diário, abr. 2020b. Disponível em: $<$ https://portalarquivos.saude.gov.br/images/pdf/20 20/April/03/BE6-Boletim-Especial-do-COE.pdf $>$

BRASIL. MINISTÉRIO DA SAÚDE (BR). Ministério da Saúde passa a testar casos leves de COVID-19. 2020c. Disponível em: $<$ https://www.bio.fiocruz.br/index.php/br/noticias/1 857-ministerio-da-saude-passa-a-testar-casos-levesda-covid-19>

BUFFON, E. A. M.; PAZ, O. L. S. Geoprocessamento para mapeamento das áreas de risco de inundações: uma aplicação na sub-bacia hidrográfica do Rio Palmital, Colombo e PinhaisParaná. Revista Brasileira de Geografia Física, v. 11, n. 6, p. 2186-2200, 2018.

CAMPOS, H. S. Influenza, uma nova tsunami. Pulmão RJ, v. 14, n. 2, p. 104-108, 2005.

CASTRO, R.; LUZ, P.M.; WAKIMOTO, M. D.; VELOSO, V. G.; GRINSZTEJN, B.; PERAZZO, H. COVID-19: uma meta-análise do diagnóstico teste precisão do comercial ensaios registrado no Brasil. Braz J Infectar Dis. 2020. Disponível em $<$ https://www.bjid.org.br/en-covid-19-metaanalysis-diagnostic-test-accuracy-articuloS1413867020300295>.

CAVALCANTE, João Roberto; ABREU, Ariane de Jesus Lopes de. COVID-19 no município do Rio de Janeiro: análise espacial da ocorrência dos primeiros casos e óbitos confirmados. Epidemiol. Serv. Saúde, Brasília, v. 29, n. 3, e 2020204, 2020.

INSTITUTO BRASILERO DE GEOGRAFIA E ESTATÍSTICA - IBGE. Regiões de Influência das cidades. 2018 . Disponível em:
$<$ https://biblioteca.ibge.gov.br/visualizacao/livros/li v101728.pdf>

INSTITUTO BRASILERO DE GEOGRAFIA E ESTATÍSTICA - IBGE. IBGE cidades: Eunápolis. 2020. Disponível em: $<$ https://cidades.ibge.gov.br/brasil/ba/eunapolis/pan orama $>$

JOLY, Carolina. Especialização produtiva do território e o circuito espacial produtivo de celulose em Eunápolis - BA. 2007, 97 f. Dissertação (Mestrado em Geografia Humana) - Universidade de São Paulo, São Paulo, 2007.

JOHNS HOPKINS UNIVERSITY. Coronavirus COVID-19 Global Cases by Johns Hopkins CSSE [Internet]. Johns Hopkins University. Disponível em: <https://coronavirus.jhu.edu/map.html>

KRITSKI, A.; ALVES, D.; WERNECK, G. ZIMMERMANN, I.; SANCHEZ, M.; GALLIEZ, R.; MEDRONHO, R. Panorama da COVID-19. Rio de janeiro: Universidade Federal do Rio de Janeiro, 2020. Disponível em: <https://ufrj.br/wpcontent/uploads/legacy/imgnoticia/2020/03/notatecnica25032020.pdf>

MESQUITA, A. A.; SERRANO, R. O. P.; LUCIO, F. S. SILVA, J. K. B.; XAVIER, G. B.; MOREIRA, J. G. B. Espacialização geográfica da COVID-19 na Amazônia Sul-Ocidental: a contribuição da geografia do risco na gestão da pandemia no estado do Acre - Brasil. UÁQUIRI: PPGGEO, v.2, n.1, p.128-143, 2020.

NASCIMENTO, F. L.; PACHECO, A. E. S. D. Sistema de saúde público no brasil e a pandemia do novo coronavírus. Boletim de Conjuntura (BOCA), Boa Vista, v. 2, n. 5, p. 63-72, abr. 2020.

OLIVEIRA NETO, T.; GARCIA, T. de S. L.; SPINUSSI, E. Pandemia de COVID-19, as fronteiras pelo mundo e o transporte aéreo na Itália. Revue franco-brésilienne de géographie/Revista francobrasilera de geografia, v. 44, 2020.

SANTOS, J. P. C.; SIQUEIRA, A. S. P; PRACA, H. L. F.; ALBUQUERQUE, H. G. Vulnerabilidade a formas graves de COVID-19: uma análise intramunicipal na cidade do Rio de Janeiro, Brasil. Cad. Saúde Pública, Rio de Janeiro, v. 36, n. 5, e00075720, abr. 2020.

SUPERINTENDÊNCIA DE ESTUDOS ECONÔMICOS E SOCIAIS - SEI. Perfil dos Territórios de Identidade da Bahia. Salvador: SEI, 2015.3 v. p. (Série territórios de identidade da Bahia, v. 1). Disponível em: $<$ http://www.sei.ba.gov.br/ind ex.php?option $=$ com_content $\&$ view $=$ article $\& i d=200$ 0\&Itemid $=284>$ 
TEN-CATEN, F.; GONZALEZ-DIAS, P.; CASTRO, I.; OGAVA, R.; GIDDALURU, J.; SILVA, J. C. S.; MARTINS, F. GONÇALVES, A. N. A.; COSTA-MARTINS, A. G.; ARAUJO, J. D.; VIEGAS, A. C.; CUNHA, F. Q.; FARSKY, S.; BOZZA, F. A. LEVIN, A. S.; PANNARAJI, P. S.; SILVA, T. I.; MINOPRIO, P.; ANDRADE, B. B.; SILVA, F. P.; NAKAYA, H. I. In-depth Analysis of Laboratory Parameters Reveals the Interplay Between Sex, Age and Systemic Inflammation in Individuals with COVID-19. medRxiv, 2020. Disponível em: $<$ https://www.medrxiv.org/content/10.1101/2020.0 8.07.20170043v2\#disqus_thread $>$
THE NOVEL CORONAVIRUS PNEUMONIA EMERGENCY RESPONSE EPIDEMIOLOGY TEAM. The epidemiological characteristics of an outbreak of 2019 new coronavirus diseases (COVID-19) in China. Chin J Epidemiol, v. 41, n. 2, 145-151.

WU, Z.; MCGOOGAN, J. M. Features and important lessons from the 2019 coronavirus disease outbreak (COVID-19) in China: summary of a report of 72314 cases from the Chinese Center for Disease Control and Prevention. JAMA, v. 323, n. 13, p. 1239-1242, 2020. 